ARTICLE

Received 8 Sep 2013 | Accepted 26 Feb 2014 | Published 8 Apr 2014

DOl: $10.1038 /$ ncomms 4524

OPEN

\title{
Observation of dark states in a superconductor diamond quantum hybrid system
}

\author{
Xiaobo Zhu ${ }^{1} \uparrow$, Yuichiro Matsuzaki ${ }^{1}$, Robert Amsüsss ${ }^{1,2}$, Kosuke Kakuyanagi ${ }^{1}$, Takaaki Shimo-Oka ${ }^{3}$, \\ Norikazu Mizuochi ${ }^{3}$, Kae Nemoto ${ }^{4}$, Kouichi Semba ${ }^{1,4, \dagger}$, William J. Munro ${ }^{1} \&$ Shiro Saito ${ }^{1}$
}

The hybridization of distinct quantum systems has opened new avenues to exploit the best properties of these individual systems. Superconducting circuits and electron spin ensembles are one such example. Strong coupling and the coherent transfer and storage of quantum information has been achieved with nitrogen vacancy centres in diamond. Recently, we have observed a remarkably sharp resonance $(\sim 1 \mathrm{MHz})$ at $2.878 \mathrm{GHz}$ in the spectrum of flux qubit negatively charged nitrogen vacancy diamond hybrid quantum system under zero external magnetic field. This width is much narrower than that of both the flux qubit and spin ensemble. Here we show that this resonance is evidence of a collective dark state in the ensemble, which is coherently driven by the superposition of clockwise and counter-clockwise macroscopic persistent supercurrents flowing in the flux qubit. The collective dark state is a unique physical system and could provide a long-lived quantum memory.

\footnotetext{
${ }^{1}$ NTT Basic Research Laboratories, NTT Corporation, 3-1 Morinosato-Wakamiya, Atsugi, Kanagawa 243-0198, Japan. ${ }^{2}$ Vienna Center for Quantum Science and Technology, Atominstitut, TU Wien, 1020 Vienna, Austria. ${ }^{3}$ Graduate School of Engineering Science, Osaka University, 1-3 Machikane-yama, Toyonaka, Osaka 560-8531, Japan. ${ }^{4}$ National Institute of Informatics, 2-1-2 Hitotsubashi, Chiyoda-ku, Tokyo 101-8430, Japan. † Present addresses: Institute of Physics, Chinese Academy of Sciences, Beijing, China. (X.Z.); National Institute of Information and Communications Technology, Tokyo, Japan. (K.S.). Correspondence and requests for materials should be addressed to S.S. (email: saito.shiro@lab.ntt.co.jp).
} 
T he last decade has seen that our understanding of the principles of quantum mechanics lead to the development of technologies underpinned by it. We are at the stage where real engineering of quantum devices is taking place. Hybridization of distinct quantum systems allows us to exploit the best properties of these individual systems without their weaknesses (refs 1-3 and references within). One well-known example of such hybridized quantum systems are superconducting circuits ${ }^{4,5}$ coupled to electron spin ensembles ${ }^{6-8}$. These hybridized systems ${ }^{9-21}$ have seen many fundamental experiments taking place including for instance, the strong coupling and the coherent transfer and storage of quantum information between the superconducting circuit and an electron spin ensemble from nitrogen vacancy $(\mathrm{NV})$ centres in diamond ${ }^{13-17}$. In these experiments, the negatively charged nitrogen vacancy $\left(\mathrm{NV}^{-}\right)$ centres were generally considered as an ensemble of two-level systems where one mode of the ensemble collectively coupled to the superconducting circuit. However, the ground-state manifold of the $\mathrm{NV}^{-}$centre is in fact a three-level system, which could show phenomena beyond those available in two-levels systems.

Dark states are a particular state of a three-level atom or molecule driven by two fields, which cannot absorb or emit photons ${ }^{22,23}$. Further, dark states are not solely the province of single atoms but can be seen in ensembles of atoms as well. While such effects have generally been investigated in quantum optical systems ${ }^{24-27}$, the potential exists from them to be observed in hybrid solid-state systems. Here we are going to consider a hybrid system (Fig. 1) composed of an ensemble of $\mathrm{NV}^{-}$centres coupled with a gap-tuneable flux qubit ${ }^{28,29}$. The gap-tuneable flux qubit can be considered as a strongly coupled coherent source of single microwave photons that can be used to pump the $\mathrm{NV}^{-}$ centre ensemble. Depending on whether an external magnetic field is applied to split these two upper levels or not, the microwave photon can be absorbed by either of the upper levels or at zero external field by a coherent superposition of those levels. Next, a strain coupling between these two upper levels allows exchange of excitations in these levels and so is also a form of effective driving term. Having these two fields opens up the possibility for the observation of a collective dark state. A number of recent experiments ${ }^{13,16}$ have hinted at a fine spectroscopy feature near the $2.878-\mathrm{GHz}$ resonance but these have not been really explored. In this work we investigate its nature.

\section{Results}

Characterization. Our characterization of this hybrid system ${ }^{16}$ begins with spectroscopic measurements (shown in Fig. 2) of two physically different ensembles (A and B) with $\mathrm{NV}^{-}$density of $\sim 4.7 \times 10^{17} \mathrm{~cm}^{-3}$ and $1.1 \times 10^{18} \mathrm{~cm}^{-3}$, respectively. Both our

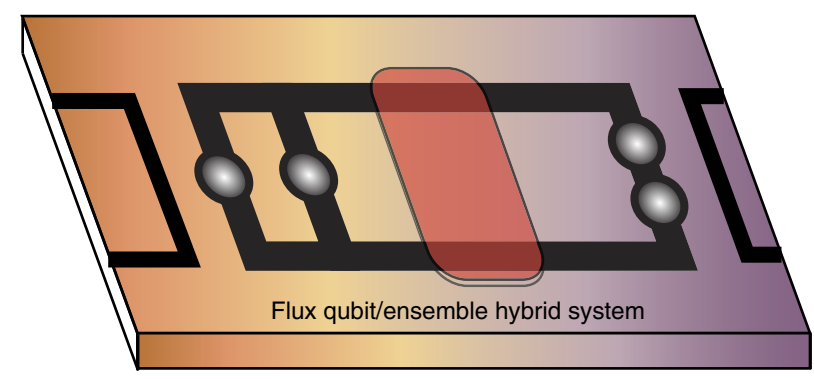

Figure 1 | Illustration of a hybrid-coupled system. The hybrid system is composed of a gap-tuneable flux qubit and an ensemble of $\mathrm{NV}^{-}$centres in diamond. The flux qubit is composed of four junctions (represented by the shaded circles). Input lines to adjust and drive the qubit are shown coming from the edges. The ensemble (light red coloured object) sits on top of the qubit. samples (Fig. 2a,c) show significant hybridization near the optimal point $\Phi_{\mathrm{qb}} \sim 1.5 \Phi_{0}$, clearly indicating strong coupling. The splitting between the hybridized peaks is $28 \mathrm{MHz}$ and $42 \mathrm{MHz}$, respectively. The energy spectrum shows a narrow peak located in the middle of the avoid crossing structure (Fig. 2a,c). To highlight this narrowness further, we show in Fig. $2 \mathrm{~d}$ a fine scan of the spectrum (c) near the centre peak. The narrowest full width at half maximum of the peak is about $1.0 \mathrm{MHz}$ (Fig. 2b) and $2.2 \mathrm{MHz}$ (Fig. 2e), respectively. This line width is much narrower than the flux qubit or ensembles natural line widths.

The model. To establish the mechanism causing this narrow central peak, we need to consider a Hamiltonian model of a driven flux qubit coupled to an ensemble of $\mathrm{NV}^{-}$centres. The full description is presented in Supplementary Note 1, but this can be significantly simplified when we consider that our flux qubit will only drive a few excitations in the ensemble. In such a situation we can model the spin ensemble as a number of harmonic oscillators ${ }^{30-32}$ and so write the Hamiltonian after a rotating wave approximation as

$$
\begin{aligned}
H= & \hbar \sqrt{\varepsilon^{2}+\Delta^{2}} \hat{\sigma}_{+} \hat{\sigma}_{-}+\hbar D \hat{b}^{\dagger} \hat{b}+\hbar D \hat{d}^{\dagger} \hat{d} \\
& +\hbar G\left(\hat{b}^{\dagger} \hat{\sigma}_{-}+\hat{\sigma}_{+} \hat{b}\right)+\hbar J\left(e^{i \theta} \hat{b}^{\dagger} \hat{d}+e^{-i \theta} \hat{d}^{\dagger} \hat{b}\right)
\end{aligned}
$$

where $\sigma \pm$ are the raising/lowering operators of the flux qubit in the energy basis $|0\rangle_{\mathrm{qb}}$ and $|1\rangle_{\mathrm{qb}}, \Delta$ represents the flux qubit's tunnel energy splitting while $\varepsilon$ is its energy bias. The ensembles are represented as two harmonic oscillators $D \hat{b}^{\dagger} \hat{b}$ and $D \hat{d}^{\dagger} \hat{d}$ where $\hat{b}^{\dagger}=\frac{1}{\sqrt{N}} \sum_{k}\left|A_{+}\right\rangle_{\mathrm{NV}_{k}}\langle 0|$ and $\hat{d}^{\dagger}=\frac{1}{\sqrt{N}} \sum_{k}\left|A_{-}\right\rangle_{\mathrm{NV}_{k}}\langle 0|$ with $\left|A_{ \pm}\right\rangle_{\mathrm{NV}_{k}}=(1 / \sqrt{2})\left[|1\rangle_{\mathrm{NV}_{k}} \pm|-1\rangle_{\mathrm{NV}_{k}}\right] . D / 2 \pi \simeq 2.878 \mathrm{GHz}$ is the mean zero-field splitting between the lowest energy level of the $\mathrm{NV}^{-}$centres and the two excited states. The term $J\left(e^{i \theta} \hat{b}^{\dagger} \hat{d}+e^{-i \theta} \hat{d}^{\dagger} \hat{b}\right)$ couples the two harmonic oscillators together allowing exchange of excitations between them. It is in effect a direct current (DC) driving term whose magnitude can be varied by detuning the $\hat{b}^{\dagger} \hat{b}$ and $\hat{d}^{\dagger} \hat{d}$ harmonic oscillators energy levels. The term $J$ arises from both strain and Zeeman effects. The flux qubit couples only to one mode of this ensemble, which we describe by $G\left(\hat{b}^{\dagger} \hat{\sigma}_{-}+\hat{\sigma}_{+} \hat{b}\right)$. It represents the flux qubit driving the ensemble with a single excitation. The coupling strength $G$ of this interaction is given by $G=\sqrt{N} g_{e} \mu_{B} B_{\mathrm{qb}}^{\perp} \Delta / \sqrt{\varepsilon^{2}+\Delta^{2}}$, where $N$ is the number of centres in the coupled ensemble, $g_{e} \mu_{B} /$ $2 \pi=28 \mathrm{MHz} \mathrm{mT}^{-1}$ and $B_{\mathrm{qb}}^{\perp}$ is the perpendicular component to the NV axis of the magnetic field generated by the flux qubit. This coupling strength can be tuned by moving the flux qubit offresonance with the ensemble (non-zero $\varepsilon$ ).

Energy levels and their associated states. Tuning the energy gap of the flux qubit $(\Delta)$ to $D$, we can straightforwardly determine the eigen energies and eigenstates of the hybrid system using equation (1). We choose for simplicity $\theta=\pi / 2$ to illustrate this (in Supplementary Note 1, we relax this constraint). The eigen energies are plotted against $J$ in Fig. 3a. The lowest energy level labelled by L 0 corresponds to the ground state $|0\rangle_{\mathrm{gb}}|0\rangle_{\text {ens }}$ where $|0\rangle_{\text {ens }}=|0\rangle_{\mathrm{NV}_{1}}|0\rangle_{\mathrm{NV}_{2}} \cdots|0\rangle_{\mathrm{NV}_{\mathrm{N}}}$. The energy level $\mathrm{L}-(\mathrm{L}+)$ corresponds to the single-excitation dressed bright states

$$
\left|\mathcal{B}_{ \pm}^{(1)}\right\rangle=\left(\frac{1}{\sqrt{2\left(G^{2}+J^{2}\right)}}\left(J \hat{d}^{\dagger}+i G \hat{\sigma}_{+}\right) \pm \frac{i}{\sqrt{2}} \hat{b}^{\dagger}\right)|0\rangle_{\mathrm{qb}}|0\rangle_{\mathrm{ens}} .
$$

The transitions between $|0\rangle_{\mathrm{qb}}|0\rangle_{\text {ens }} \leftrightarrow\left|\mathcal{B}_{ \pm}^{(1)}\right\rangle$ corresponds to the two broader peaks shown in Fig. $2 \mathrm{a}, \mathrm{c}$ near $\Phi_{\mathrm{qb}} \sim 1.5 \Phi_{0}$. These transitions are being directly driven. 

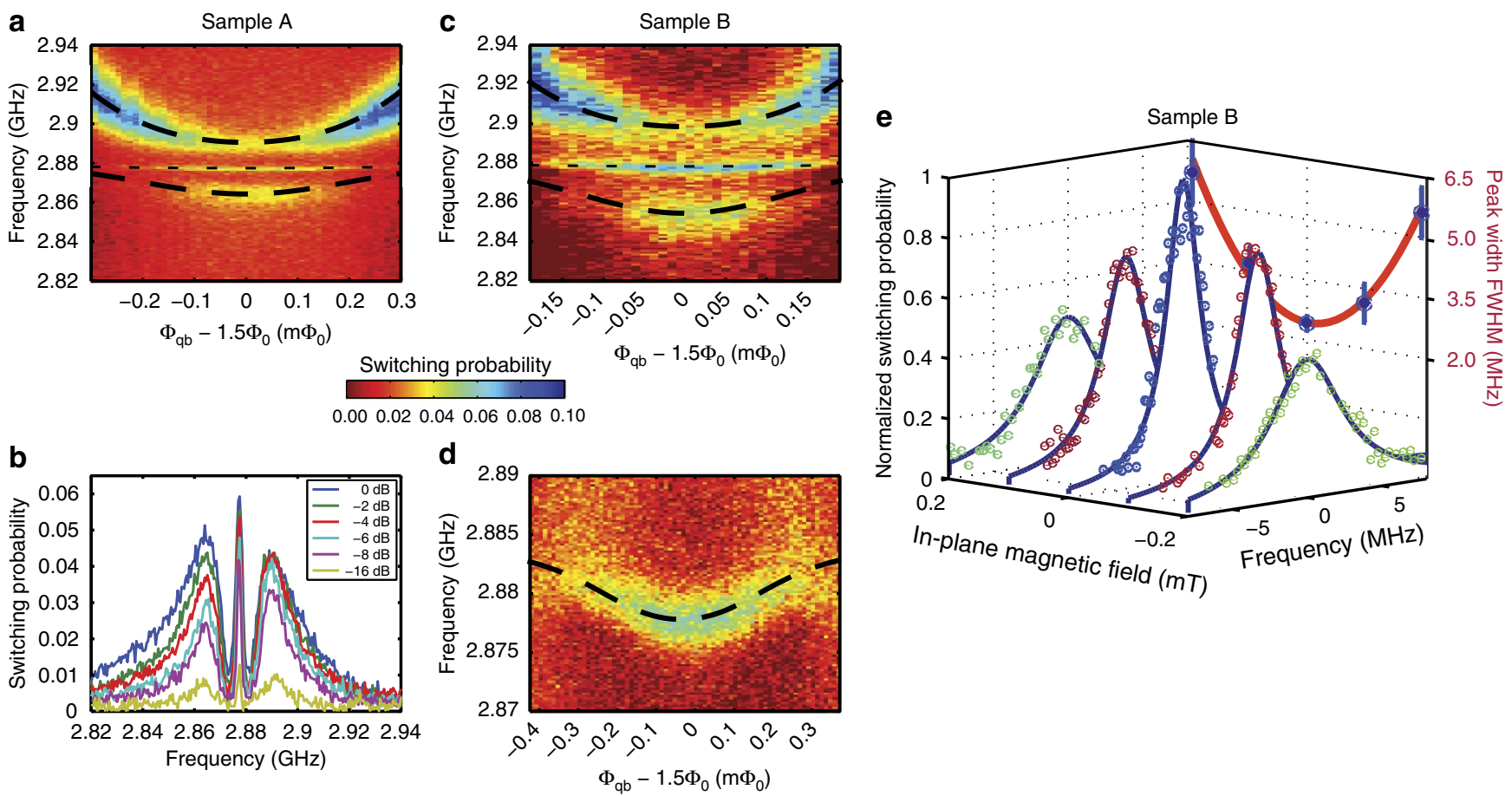

Figure 2 | Spectroscopy characterization of the hybrid system. Energy spectrum of a gap-tuneable flux qubit coupled separately to two different density $\mathrm{NV}^{-}$spin ensembles (samples $\mathrm{A}$ and $\mathrm{B}$ ), the first having $4.7 \times 10^{17} \mathrm{~cm}^{-3}(\mathbf{a}, \mathbf{b})$ and the second $1.1 \times 10^{18} \mathrm{~cm}^{-3}$ (c-e). The peak splittings were determined as $28 \mathrm{MHz}$ for sample $\mathrm{A}$ and $42 \mathrm{MHz}$ for sample B. Both spectroscopic measurements a and c show a sharp peak located in the middle of the avoided crossing structure. In $\mathbf{b}$ we show the microwave power dependence of the spectrum for a fixed flux bias $\left(\Phi_{\mathrm{qb}}=1.5 \Phi_{0}\right)$. The centre sharp peak exists for all probe powers and shows a higher amplitude than the outer two broader peaks implying it is unlikely to result from a two-photon/ nonlinear process. At the pump power used, our flux qubit would normally show power-broadening effects. In d, a fine scan of the spectrum (c) near the centre peak part is illustrated. The sharp peak shows a small flux bias $\Phi_{\mathrm{qb}}$ dependence $\left(\Phi_{\mathrm{qb}}\right.$ is the flux through the qubit loop). The eigen energies (black dashed lines) of the spectrums $\mathbf{a}, \mathbf{c}$ and $\mathbf{d}$ can be reproduced by diagonalizing the full Hamiltonian of the system (Supplementary Note 1). In $\mathbf{e}$, the normalized switching probability of the central peak is shown for several small in-plane magnetic fields (our diamond crystal was attached on top of the superconducting circuit with the (001) surface facing the flux qubit). The small in-plane magnetic field splits the degenerate excited states $| \pm 1\rangle$ of the $\mathrm{NV}^{-}$ensemble weakening the effect of the strain interaction. The larger the splitting, the weaker the interaction. The full width at half maximum of the peak is minimal at zero in-plane magnetic field and becomes larger when the amplitude of the magnetic field increases. Spectra $\mathbf{a}$ and $\mathbf{b}$ were measured with a 0-mT in-plane magnetic field while $\mathbf{c}$ and $\mathbf{d}$ were measured with a small $-0.1 \mathrm{mT}$ in-plane magnetic field. The error bars represent the $95 \%$ confidence interval while fitting to the resonance peaks.

a

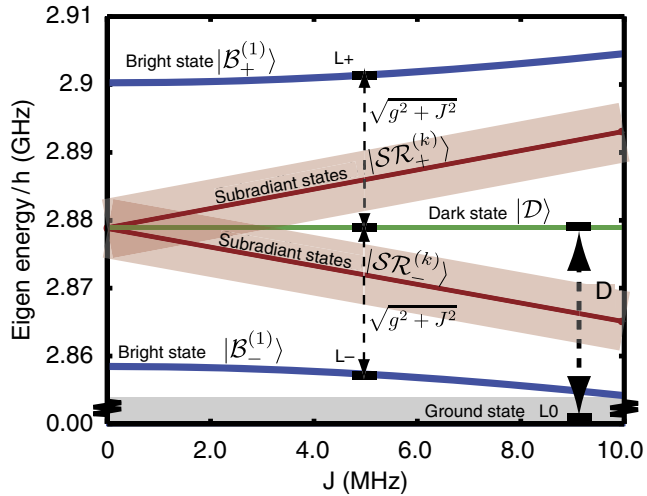

b

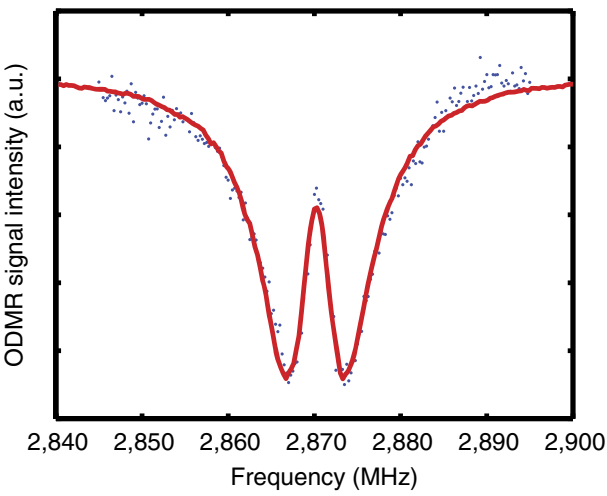

Figure 3 | Energy-level diagram of the coupled system. In a, we illustrate the energy-level diagram for the superconducting flux qubit-NV ${ }^{-}$centre ensemble as a function of the $J$ at the optimal point $\Phi_{\mathrm{qb}}=1.5 \Phi_{0}$. The energy levels for the bright states are labelled by $\left|\mathcal{B}_{ \pm}^{(1)}\right\rangle$ while the dark state $|\mathcal{D}\rangle$ energy is seen at $2.878 \mathrm{GHz}$. There are many subradiant states $\left|\mathcal{S} \mathcal{R}_{ \pm}^{(k)}\right\rangle$ that can be broadened significantly by strain variation and inhomogenous broadening (light red). In $\mathbf{b}$, the optically detected magnetic resonance spectrum of the $\mathrm{NV}^{-}$ensemble is given (at room temperature). The two excited states at the $k$ th spin wave are energetically separated by $J$. The blue dots denotes the experimentally measured data while the red line shows our numerically simulated results (described in Supplementary Note 2). With the room temperature measurement $D / 2 \pi \sim 2.87 \mathrm{GHz}$ while at the low temperature the zero-field splitting increases to $D / 2 \pi \sim 2.878 \mathrm{GHz}$ (refs $13,16,36$ ). 
Next in our diagram are energy levels associated with the subradiant states. These are composed of states having only a single excitation in the ensemble. These states have no $|1\rangle_{\mathrm{gb}}|0\rangle_{\text {ens }}$ component and so cannot be detected using flux qubit spectroscopic measurements. For finite $J$, these subradiant states are split either above or below the $2.878-\mathrm{GHz}$ resonance (Fig. 3a) and in the limit $g_{e} \mu_{B} B_{\mathrm{qb}}^{\perp} \ll J$ be written as the spin waves $(k>0)$

$$
\begin{aligned}
\left|\mathcal{S R}_{ \pm}^{(k)}\right\rangle & \simeq \frac{1}{\sqrt{N}}\left\{\sum_{j=1}^{N} e^{i 2 \pi(j-1) k / N}\left|A_{ \pm}\right\rangle_{\mathrm{NV}_{j}}\langle 0|\right\}|0\rangle_{\mathrm{ens}} \\
& \equiv\left|\mathcal{W}_{ \pm}^{(k, k \neq 0)}\right\rangle
\end{aligned}
$$

with an energy of $E_{\mathcal{S R}_{ \pm}^{(k)}} \sim D \pm J$. These spin waves are orthogonal to the $k=0$ mode and so do not couple to the flux qubit.

Finally, in terms of single excitation energy levels is the eigenstate at $2.878 \mathrm{GHz}$, which corresponds to

$$
|\mathcal{D}\rangle=\left(\frac{G}{\sqrt{G^{2}+J^{2}}} \hat{d}^{\dagger}-\frac{i J}{\sqrt{G^{2}+J^{2}}} \hat{\sigma}_{+}\right)|0\rangle_{\mathrm{qb}}|0\rangle_{\mathrm{ens}} .
$$

This state can further be written in the form $|\mathcal{D}\rangle=$ $\left(G /\left(\sqrt{G^{2}+J^{2}}\right)\right)|0\rangle_{\mathrm{qb}}\left|\mathcal{W}_{-}^{(k=0)}\right\rangle-\left(i J /\left(\sqrt{G^{2}+J^{2}}\right)\right)|1\rangle_{\mathrm{qb}}|0\rangle_{\mathrm{ens}}$ where $\left|\mathcal{W}_{-}^{(k=0)}\right\rangle=(1) /(\sqrt{N})\left\{\sum_{i=1}^{N}\left|A_{-}\right\rangle_{\mathrm{NV}_{j}}\langle 0|\right\}|0\rangle_{\text {ens }}$. The form of dark state $|\mathcal{D}\rangle$ is important because it contains both components of an excitation in the flux qubit $|1\rangle_{\mathrm{q} b}|0\rangle_{\text {ens }}$ and the ensemble. The component $|1\rangle_{\mathrm{qb}}|0\rangle_{\text {ens }}$ implies that a signal will be seen at $2.878 \mathrm{GHz}$ in the spectroscopic measurements (observed in Fig. 2). Next, as we tune the flux qubit away from the resonance point using the energy bias $\varepsilon$, we would expect to see a slight quadratic $\Phi_{\mathrm{gb}}$ dependence in the observed resonance frequency (Fig. 2d) owing to the small $|1\rangle_{\mathrm{qb}}|0\rangle_{\text {ens }}$ component in $|\mathcal{D}\rangle$. When we apply an in-plane magnetic field, we effectively strengthen the driving field $J$ and so we increase the contribution of the $|1\rangle_{\mathrm{qb}}|0\rangle_{\mathrm{ens}}$ component to $|\mathcal{D}\rangle$. The population of this component is proportional to $J$ and so we should expect to observe a quadratic dependance in the observed line width (observed in Fig. 2e). We can also understand the powerdependent measurements in (Fig. 2b). Given that the $J$ field is not changing during these experiments, we would expect the area of the central peak to decrease at approximately the same linear rate as the outer broader peaks as the pump field driving the flux qubit decreases. This is clearly what is seen even at very low pump powers (Fig. 4) and clearly shows this is not a two-photon process. The amplitude of the centre peak being higher than the outer peaks is also an indicator.

Coherence properties. The line width of these peaks depends on the coherence properties of our hybrid system. Surprisingly, the line width of the central peak, $1.0 \mathrm{MHz}$ for sample $\mathrm{A}$ and $2.2 \mathrm{MHz}$ for sample B, seems quite narrow compared with the usual coherence properties of the system. The measured flux qubit coherence properties at the optimal point are $T_{1} \sim 300 \mathrm{~ns}$ (relaxation), $T_{2} \sim 150 \mathrm{~ns}$ (dephasing) ${ }^{16}$. However, in the spectroscopic measurements, power broadening of $\sim 4 \mathrm{MHz}$ makes these much worse. Further, the coherence properties also decrease rapidly as we move away from the optimal point. As $|\mathcal{D}\rangle$ is mainly composed of the state $|0\rangle_{\mathrm{qb}}\left|\mathcal{W}_{-}^{(k=0)}\right\rangle$, it is now easy to understand why the dark states' line width does not change much as the flux qubit moves away from the optimal point (by increasing $\varepsilon$ ). Although the flux qubit line width increases, it has a minimal contribution to $|\mathcal{D}\rangle$ (proportionally to $J^{2} /\left(G^{2}+J^{2}\right)$ ). Next, the electron spin ensemble $T_{1}^{(\mathrm{NV})}$ has
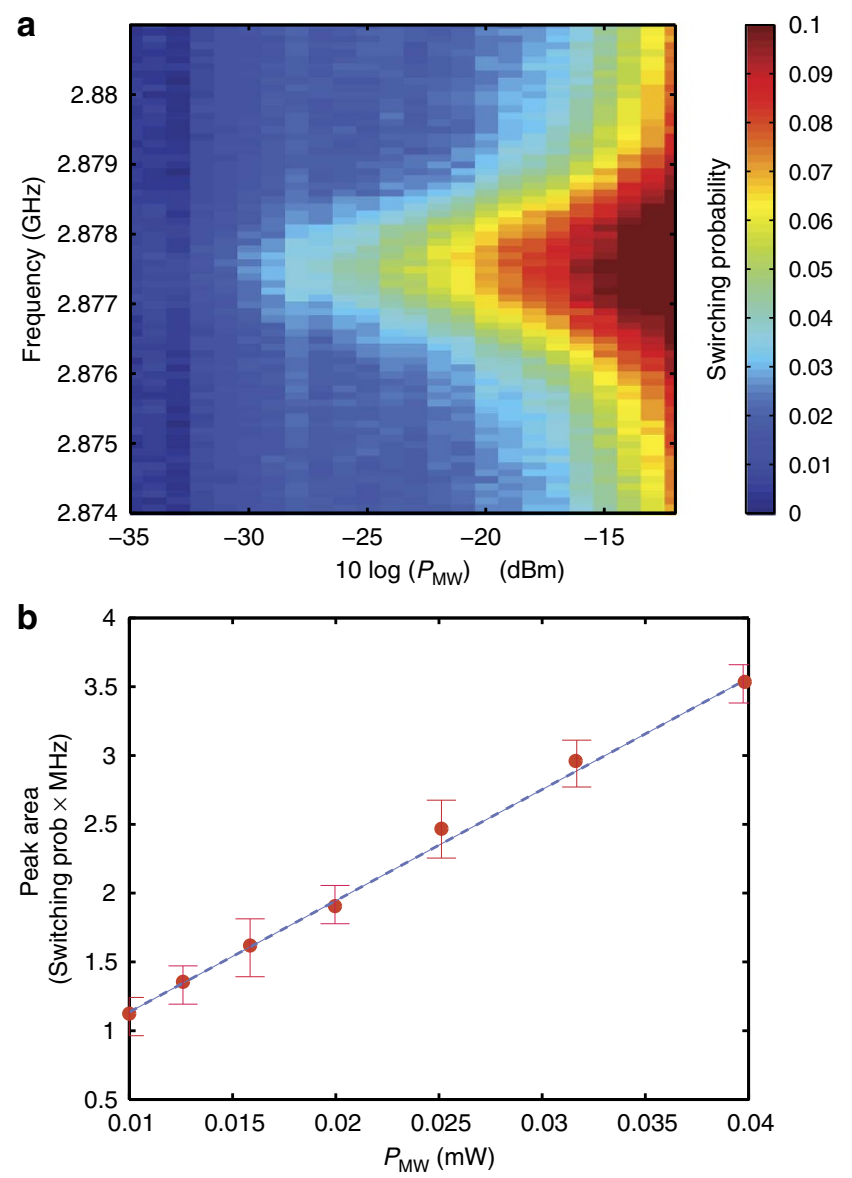

Figure 4 | Power dependence of the central peak of sample A. In a, we show the energy spectrum of the gap-tuneable flux qubit coupled separately the $\mathrm{NV}^{-}$spin ensemble for varying microwave pump power $P_{\mathrm{MV}}$ $(\mathrm{mW})$ at room temperature. In $\mathbf{b}$, the area of the central peak is shown against $P_{\mathrm{MV}}(\mathrm{mW})$. The error bars represent the $95 \%$ confidence interval while fitting to the resonance peaks. The dashed line represents a simple linear fit to the data points.

been measured to be $\sim 40 \mathrm{~s}$ (ref. 15); however, $T_{2}^{*}$ is much shorter (tens of nanoseconds) in our case ${ }^{17}$. Inhomogenous broadening due to random magnetic fields caused by nitrogen (P1) and other centres in the diamond sample A (B) is likely to be the primary cause of this and it has been derived from the fitting to the spectrum data at $\sim 3.1 \mathrm{MHz}(7.8 \mathrm{MHz}$; see Supplementary Notes 1 and 2). The distribution of the strain field is $\sim 4.4 \mathrm{MHz}$ $(7.6 \mathrm{MHz})$ while fluctuations in the zero-field splitting $D$ are $0.45 \mathrm{MHz}(0.75 \mathrm{MHz})$. An always-on hyperfine coupling to thermalized nitrogen nuclear spins is $\sim 2.3 \mathrm{MHz}$ (ref. 17). These values are consistent with the previous experimental results ${ }^{16,17}$. The measured line width of the central peak is narrower than all these (apart from $D$ fluctuations). Our modelling of these system indicates that this central peaks is not limited by the effects of the strain field or inhomogenous broadening. Instead, it seems currently limited by qubit power broadening and zero-field fluctuations.

\section{Discussion}

The energy diagram in (Fig. 3a) illustrates why this collective state has such a narrow line width (and hence long lifetime). The state $|\mathcal{D}\rangle$ would couple with other subradiant states and dephase quickly if a significant number of them existed near $2.878 \mathrm{GHz}$. 
Fortunately, the existence of inhomogenous broadening and the strain field induces an energy shift from $2.878 \mathrm{GHz}$ on the subradiant states leaving this collective dark state well isolated. Since the dephasing process is induced by low-frequency noise, such an energy gap can suppress unwanted transitions between $(|\mathcal{D}\rangle)$ and environmental states $\left(\left|\mathcal{S} \mathcal{R}_{ \pm}^{(k)}\right\rangle\right)$. This is quite different from what happens with general atom-based dark states. We have simulated the effects of strain variation and inhomogenous broadening using the full Hamiltonian (detailed in Supplementary Note 1) along with decoherence on the flux qubit, enabling us to confirm our analytical results described above. Moreover, the room temperature optically detected magnetic resonance spectrum measurements ${ }^{33-35}$ support our interpretation that our subradiant states' energies are sparsely occupied near the central peak in Fig. 3b. At room temperature, this reduction in population is at $2.87 \mathrm{GHz}$ but shifts to $2.878 \mathrm{GHz}$ at $\mathrm{mK}$ temperatures ${ }^{36}$. Thus, we have strong evidence for the existence of this collective dark state.

Generally with dark states, if one turns off the control field, the dark state disappears. In our situation, here we cannot turn off the DC $J$ field but by applying a large magnetic field we can split the $| \pm 1\rangle$ levels by nearly $100 \mathrm{MHz}$. The coupling field is then effectively off-resonance because of the energy splitting. Experimentally, only double peaks showing the hybridization are seen either resonant with the $|0\rangle \leftrightarrow|+1\rangle$ transition or the $|0\rangle \leftrightarrow|-1\rangle$ transition. No fine feature is seen at $2.878 \mathrm{GHz}$. This is further evidence of our collective dark state (in Supplementary Note 3 where we show how the observed phenomenon is inconsistent with an ensemble of two-level systems).

To summarize, we have experimentally observed a narrow peak in the avoided crossing structure of the energy spectrum in the flux qubit $\mathrm{NV}^{-}$diamond hybrid system. Our analysis indicates that this is the signature of a collective dark state, which should be long lived and could provide an alternative approach for quantum memories. The lifetime of this peak can further be increased in the near future with higher quality diamond samples that possess a smaller number of nitrogen centres and that have reduced homogenous broadening and zero-field fluctuations. This can be partially achieved by using electron beam irradiation to form the $\mathrm{NV}^{-}$centres.

\section{Methods}

Measurements. Spectroscopic measurements were achieved by initializing the hybrid system in its ground state through relaxation. A 1- $\mu$ s-long microwave pulse was applied to the flux qubit followed immediately by a current pulse to the DC-superconducting quantum interference device for detecting the state of the qubit. By repeating the measurement several thousand times, we obtain the switching probability $P_{\mathrm{sw}}$, which is proportional to the qubit excited-state population.

\section{References}

1. Wallquist, M., Hammerer, K., Rabl, P., Lukin, M. \& Zoller, P. Hybrid quantum devices and quantum engineering. Phys. Scr. T137, 014001 (2009).

2. Duty, T. Towards superconductor-spin ensemble hybrid quantum systems. Physics 3, 80 (2010).

3. Xiang, Z., Ashhab, S., You, J. Q. \& Nori, F. Hybrid quantum circuits: superconducting circuits interacting with other quantum systems. Rev. Mod. Phys. 85, 623-653 (2013).

4. Clarke, J. \& Wilhelm, F. K. Superconducting quantum bits. Nature 453, 1031-1042 (2008)

5. Devoret, M. H. \& Schoelkopf, R. J. Superconducting circuits for quantum information: an outlook. Science 339, 1169-1174 (2013).

6. Brion, E., Molmer, K. \& Saffman., M. Quantum computing with collective ensembles of multilevel systems. Phys. Rev. Lett. 99, 260501 (2007).

7. Wu, H. et al. Storage of multiple coherent microwave excitations in an electron spin ensemble. Phys. Rev. Lett. 105, 140503 (2010).

8. Wrachtrup, J. \& Jelezko, F. Processing quantum information in diamond. J. Phys. Condens. Matter 18, S807 (2006).
9. Verdú, J. et al. Strong magnetic coupling of an ultracold gas to a superconducting waveguide cavity. Phys. Rev. Lett. 103, 043603 (2009).

10. Imamoglu, A. Cavity QED based on collective magnetic dipole coupling: spin ensembles as hybrid two-level systems. Phys. Rev. Lett. 102, 083602 (2009).

11. Wesenberg, J. H. et al. Quantum computing with an electron spin ensemble. Phys. Rev. Lett. 103, 070502 (2009).

12. Schuster, D. I. et al. High-cooperativity coupling of electron-spin ensembles to superconducting cavities. Phys. Rev. Lett. 105, 1405011 (2010).

13. Kubo, Y. et al. Strong coupling of a spin ensemble to a superconducting resonator. Phys. Rev. Lett. 105, 140502 (2010).

14. Kubo, Y. et al. Hybrid quantum circuit with a superconducting qubit coupled to a spin ensemble. Phys. Rev. Lett. 107, 220501 (2011).

15. Amsüss, R. et al. Cavity QED with magnetically coupled collective spin states. Phys. Rev. Lett. 107, 060502 (2011).

16. Zhu, X. et al. Coherent coupling of a superconducting flux-qubit to an electron spin ensemble in diamond. Nature 478, 221-224 (2011).

17. Saito, S. et al. Towards realizing a quantum memory for a superconducting qubit: Storage and retrieval of quantum states. Phys. Rev. Lett. 111, 107008 (2013).

18. Probst, S. et al. Anisotropic rare-earth spin ensemble strongly coupled to a superconducting resonator. Phys. Rev. Lett. 110, 157001 (2013).

19. Bushev, P. et al. Ultralow-power spectroscopy of a rare-earth spin ensemble using a superconducting resonator. Phys. Rev. B 84, 060501 (2011).

20. Abe, E., Wu, H., Ardavan, A. \& Morton, J. J. Electron spin ensemble strongly coupled to a three-dimensional microwave cavity. Appl. Phys. Lett. 98, 251108 (2011).

21. Chiorescu, I., Groll, N., Bertaina, S., Mori, T. \& Miyashita, S. Magnetic strong coupling in a spin-photon system and transition to classical regime. Phys. Rev. B 82, 024413 (2010).

22. Fleischhauer, M., Imamoglu, A. \& Marangos, J. P. Electromagnetically induced transparency: optics in coherent media. Rev. Mod. Phys. 77, 633-673 (2005).

23. Boller, K. J., Imamoglu, A. \& Harris, S. E. Observation of electromagnetically induced transparency. Phys. Rev. Lett. 66, 2593-2596 (1991).

24. Schmidt, H. \& Imamoglu, A. Giant Kerr nonlinearities obtained by electromagnetically induced transparency. Opt. Lett. 21, 1936-1938 (1996).

25. Hau, L. V., Harris, S. E., Dutton, Z. \& Behroozi, C. H. Light speed reduction to 17 metres per second in an ultracold atomic gas. Nature 397, 594-598 (1999).

26. Mucke, M. et al. Electromagnetically induced transparency with single atoms in a cavity. Nature $465,755-758$ (2010)

27. Fleischhauer, M. \& Lukin, M. D. Dark-state polaritons in electromagnetically induced transparency. Phys. Rev. Lett. 84, 5094-5097 (2000).

28. Paauw, F. G., Fedorov, A., Harmans, C. J. P. M \& Mooij, J. E. Tuning the gap of a superconducting flux qubit. Phys. Rev. Lett. 102, 090501 (2009).

29. Zhu, X., Kemp, A., Saito, S. \& Semba, K. Coherent operation of a gap-tunable flux qubit. Appl. Phys. Lett. 97, 102503 (2010).

30. Marcos, D. et al. Coupling nitrogen-vacancy centers in diamond to superconducting flux qubits. Phys. Rev. Lett. 105, 210501 (2010).

31. Muller, C., Shnirman, A. \& Makhlin, Y. Relaxation of Josephson qubits due to strong coupling to two-level systems. Phys. Rev. B 80, 134517 (2009).

32. Diniz, I. et al. Strongly coupling a cavity to inhomogeneous ensembles of emitters: potential for long-lived solid-state quantum memories. Phys. Rev. A 84, 063810 (2011).

33. Davies, G. Properties and Growth of Diamond (IEE/INSPEC, 1994).

34. Gruber, A. et al. Scanning confocal optical microscopy and magnetic resonance on single defect centers. Science 276, 2012-2014 (2007).

35. Mizuochi, N. et al. Coherence of single spins coupled to a nuclear spin bath of varying density. Phys. Rev. B 80, 041201R (2009).

36. Doherty, M. W. et al. The temperature shifts of the resonances of the $\mathrm{NV}^{-}$ center in diamond. Preprint at http://arxiv.org/abs/1310.7303 (2013).

\section{Acknowledgements}

We thank H. Tanji-Suzuki, K. Azuma, H. Nakano, M. Koashi, J. Schmiedmayer, J. Mayer, M.S. Everitt and A. Kemp for valuable discussions during this work. This research is granted by the Japan Society for the Promotion of Science (JSPS) through the Funding Program for World-Leading Innovative R\&D on Science and Technology (FIRST Program), initiated by the Council for Science and Technology Policy (CSTP). This research was also executed under the Commissioned Research of National Institute of Information and Communications Technology (NICT), Japan. R.A. was supported by the Doctoral Programme CoQuS (W1210)

\section{Author contributions}

All authors contributed extensively to the work presented in this paper. X.Z., R.A and S.S. carried out measurements and data analysis on the coupled flux qubit/NV ensemble. T.S., N.M. and K.S. prepared and characterized the $\mathrm{NV}^{-}$diamond crystals. X.Z. and S.S. designed and fabricated the flux qubit and associated devices while S.S. and K.K. designed and developed the flux qubit measurement system. Y.M., K.N. and W.J.M. 
provided theoretical support and analysis. X.Z., W.J.M., Y.M. and S.S. wrote the manuscript, with feedback from all authors. K.S., W.J.M. and S.S. supervised the project.

\section{Additional information}

Supplementary Information accompanies this paper at http://www.nature.com/ naturecommunications

Competing financial interests: The authors declare no competing financial interests.

Reprints and permission information is available online at http://npg.nature.com/ reprintsandpermissions/
How to cite this article: Zhu, X. et al. Observation of dark states in a superconductor diamond quantum hybrid system. Nat. Commun. 5:3524 doi: 10.1038/ncomms4524 (2014).

(c) (1) (2) This work is licensed under a Creative Commons Attributionc. ${ }_{\text {BY NC SA }}$ NonCommercial-ShareAlike 3.0 Unported License. The images or other third party material in this article are included in the article's Creative Commons license, unless indicated otherwise in the credit line; if the material is not included under the Creative Commons license, users will need to obtain permission from the license holder to reproduce the material. To view a copy of this license, visit http:// creativecommons.org/licenses/by-nc-sa/3.0/ 


\section{Publisher Correction: Observation of dark states in a superconductor diamond quantum hybrid system}

Xiaobo Zhu, Yuichiro Matsuzaki, Robert Amsüss, Kosuke Kakuyanagi, Takaaki Shimo-Oka, Norikazu Mizuochi, Kae Nemoto, Kouichi Semba, William J. Munro \& Shiro Saito

Nature Communications 5:3524 doi: 10.1038/ncomms4524 (2014); Published 8 Apr 2014; Updated 29 Mar 2018

The original HTML version of this Article had an incorrect article number of 3424; it should have been 3524 . This has now been corrected in the HTML; the PDF version of the Article was correct from the time of publication.

\footnotetext{
(c) (i)

Open Access This article is licensed under a Creative Commons Attribution 4.0 International License, which permits use, sharing, adaptation, distribution and reproduction in any medium or format, as long as you give appropriate credit to the original author(s) and the source, provide a link to the Creative Commons license, and indicate if changes were made. The images or other third party material in this article are included in the article's Creative Commons license, unless indicated otherwise in a credit line to the material. If material is not included in the article's Creative Commons license and your intended use is not permitted by statutory regulation or exceeds the permitted use, you will need to obtain permission directly from the copyright holder. To view a copy of this license, visit http://creativecommons.org/licenses/by/4.0/
}

(C) The Author(s) 2018 